\title{
Changes in the Shape of the Photoplethysmographic Signal in Response to the Active Orthostatic Test
}

\author{
Mateusz Pałasz, Marek Żyliński, Gerard Cybulski \\ Institute of Metrology and Biomedical Engineering, Department of Mechatronics, \\ Warsaw University of Technology, Warsaw, Poland
}

\begin{abstract}
The aim of the study was to check if the shape of photopletysmographic signal (PPG) is modified by the active orthostatic maneuver. We hoped to verify if such a preliminary analysis could provide any information on the condition of the cardiovascular system and its regulation.

We recorded simultaneously ECG (as a reference gating signal) and PPG. Signals were sampled at the rate of $1000 \mathrm{~Hz}$ in 6 male and 1 female subjects, who changed position from supine to standing (remaining 5 minutes in each). We used the indices of the shape of PPG signals known from literature area under the curve (AUC), rise time (RT) and proposed two other: the time of the maximum derivative occurrence on ascending slope (Tmax) -time of occurrence of the minimal derivative on descending slope (Tmin) of the plethysmographic curve.

The indices obtained in two positions were compared using t-Student test. It was found that RT, AUC and Tmax, were significantly higher in standing position than in supine, whereas Tmin was lower. Basing on these very preliminary results we could conclude that some indices characterizing the shape of the PPG signal are modified by the changing of the position of subject. We could only speculate that such indices might be used for noninvasive assessment of cardiovascular system.
\end{abstract}

\section{Introduction}

The high incidence of the cardiovascular diseases and its life-critical nature require quick, easily accessible, and useful diagnostics. Both electrocardiography, which is helpful in diagnostic for many years, and increasingly used photopletysmographic signal (PPG) can provide important information on the condition of the cardiovascular system.

Photoplethysmography is very promising technique [1]. PPG was applied in diagnose of coronary heart disease [2], childhood pneumonia [3] in assessment of cardiovascular risk $[4,5]$. PPG can be used to monitoring patient condition during sleep [6].

The aim of the study was to check if the shape of photopletysmographic signal is modified by the active orthostatic maneuver.

\section{Material and methods}

\subsection{Subjects and measurement protocol}

6 male and 1 female subject participated in this study (average age of subject was 32 years with standard deviation of 11 years; mean height was $180 \pm 5 \mathrm{~cm}$ and weight was $80 \pm 16 \mathrm{~kg}$ ). None of the subjects had diagnosed the symptoms of cardiovascular disease already diagnosed. Subjects performed 4 actives orthostatic maneuvers separated by a 5-minute rest in a respective position (supine or standing). Subject begin test from laying.

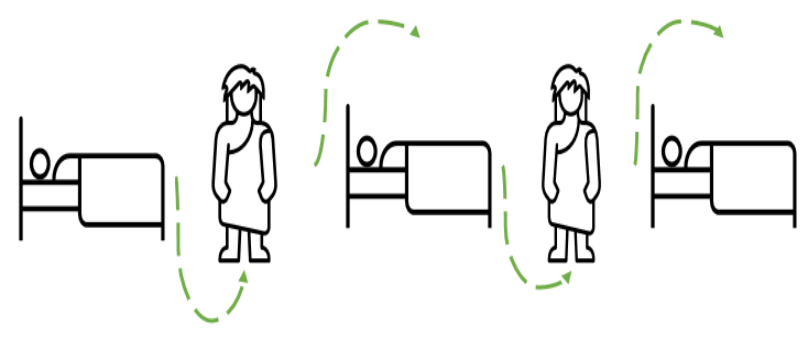

Figure 1. Measurement protocol scheme; subject performed four orthostatic maneuvers: two times gets-up and two times lies down.

\subsection{Measurement equipment}

ECG (AD Instruments Octal Bio Amp FE238) and photopletysmographic signal (Nonin 7500 Pulse Oximeter) were simultaneously recorded using AD Instruments PowerLab 16/35 DAQ at sampling frequency of $1000 \mathrm{~Hz}$.

The equipment used for PPG and ECG signal acquisition is presented on Figure 2.

\subsection{Signal analysis}

Signals analysis was performed in Sigman application (https://github.com/k-cybulski/sigman-project/).

Application was written in Python and design for multisignals analysis. The automated procedures implemented in program allow to detect $\mathrm{P}, \mathrm{D}, \mathrm{M}$ points and dicrotic notch in PPG. 


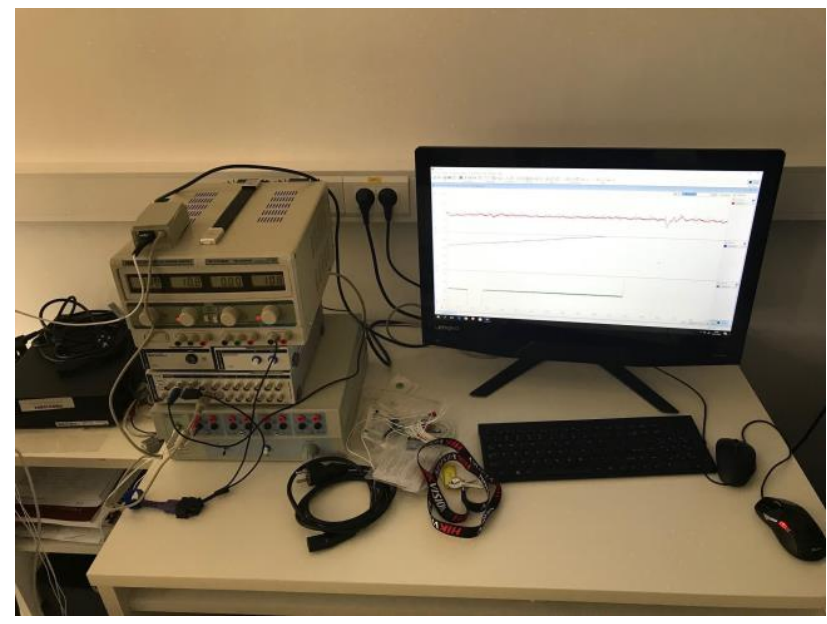

Figure 2. The equipment used for ECG and PPG signal acquisition.

Rise time (RT) was estimated as time between maximum value of the PPG signal in cardiac cycle ( $\mathrm{P}$ point) and local minimum before that point. The method of RT determination was presented on Figure 3. .

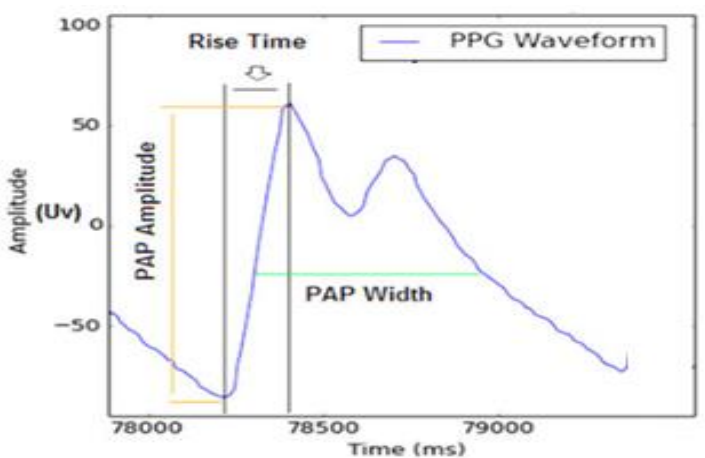

Figure 3. The method of determination of rise time (RT) index basing on the first derivative of PPG signal.

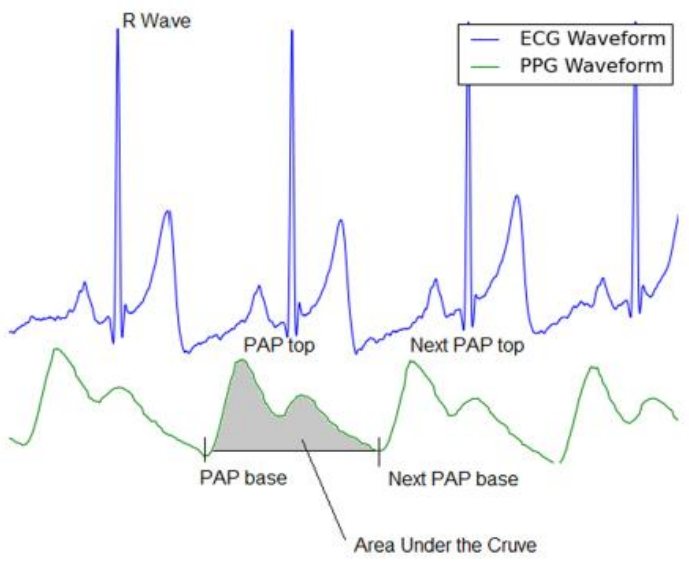

Figure 4. Area under the curve index (AUC) is an integral under the PPG trace. AUC is measured in arbitrary units.
The area under the curve index (AUC) was calculated as an integral under the PPG trace. AUC is measured in arbitrary units [au]. The method for AUC determination is presented on Figure 4.

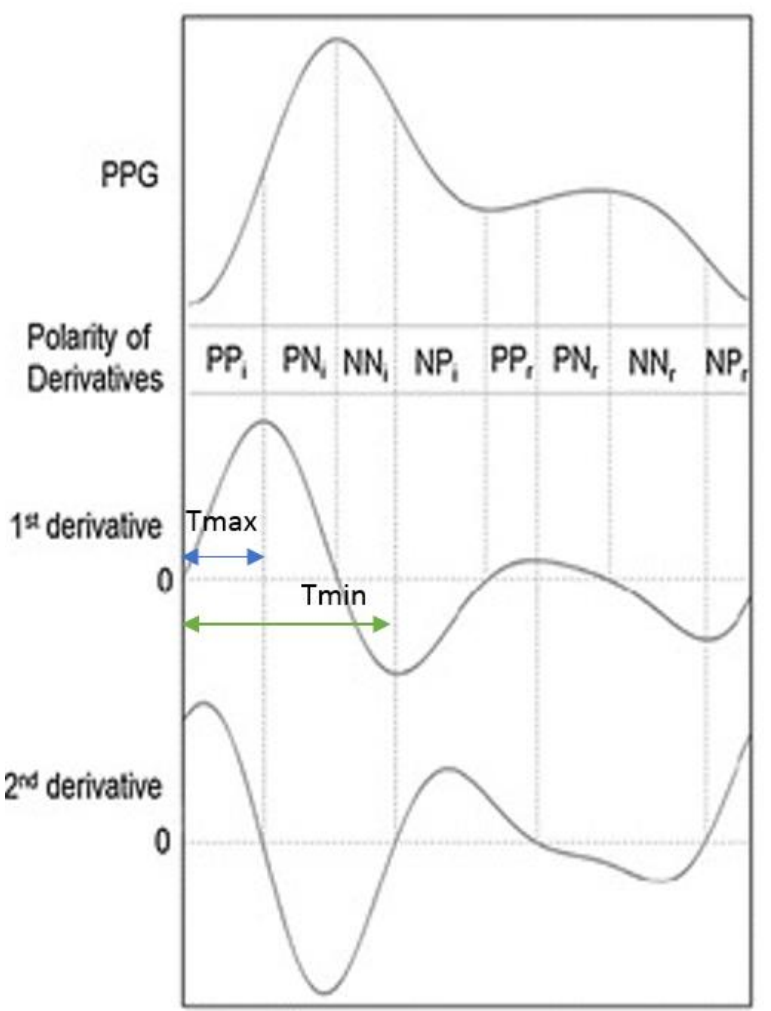

Figure 5. The method of Tmax and Tmin determination basing on the first derivative of PPG signal. Tmax as time of maximum in $1^{\text {st }}$ derivative and Tmin as time of its minimum

Tmax was estimated as the time of occurrence the maximum first derivative on ascending slope of the PPG counted from the zero value of the first derivative.

Tmin was estimated as the time of occurrence of the minimal first derivative on descending slope of PPG counted from the zero value of the first derivative.

\section{Results}

Mean values of RT, AUC, Tmax and Tmin in supine and standing for whole study population were presented in the table below. We used t-Student test to examine the differences between the mean values of respective indices. Results of that comparison is presented in Table 1.

Table 1. Differences between the mean values of indices characterising the shape of PPG signal
Supine Standing $\mathrm{p}$ 


\begin{tabular}{llll}
\hline RT [s] & 0,040 & 0,026 & $\mathrm{p}<0.00001$ \\
AUC [au] & 0,0033 & 0,0004 & $\mathrm{p}<0.00001$ \\
Tmax [s] & 0,0158 & 0,0204 & $\mathrm{p}<0.002$ \\
Tmin [s] & 0,124 & 0,114 & $\mathrm{p}<0.005$ \\
\hline
\end{tabular}

\section{Discussion and Conclusions}

We proposed two new indices of the shape of PPG signal that might be used for assessment of cardiovascular system:

-the time of the maximum derivative occurrence on ascending slope,

-the time of occurrence of the minimal derivative on descending slope of the plethysmographic curve.

We studied changes in Tmin, Tmax, RT and AUC caused by active orthostatic manoeuvres in 7 subjects.

We found statistically significant (at least $\mathrm{p}<0.05$ ) difference between mean values of all indices calculated for supine and standing position. Basing on these very preliminary results we could conclude that some indices characterizing the shape of the PPG signal are modified by the changing of the position of subject.

We could only speculate that such indices might be used for non-invasive assessment of cardiovascular system.

\section{Acknowledgements}

The study was supported by the research grants provided by Department of Mechatronics, Warsaw University of Technology.

\section{References}

[1] J. Moraes, M. Rocha, G. Vasconcelos, J. Vasconcelos Filho, V. de Albuquerque, and A. Alexandria, "Advances in Photopletysmography Signal Analysis for Biomedical Applications," Sensors, vol. 18, no. 6, p. 1894, Jun. 2018 [Online]. Available: http://dx.doi.org/10.3390/s18061894

[2] T. Otsuka, T. Kawada, M. Katsumata, C. Ibuki, Utility of second derivative of the finger photoplethysmogram for the estimation of the risk of coronary heart disease in the general population. Circulation Journal. 70, 304-310 (2006).

[3] W. Karlen, S. Raman, J. M. Ansermino, G. A. Dumont, Multiparameter respiratory rate estimation from the photoplethysmogram. IEEE Transactions on Biomedical Engineering. 60, 1946-1953 (2013).

[4] T. Otsuka, T. Kawada, M. Katsumata, C. Ibuki, Y. Kusama, Independent determinants of second derivative of the finger photoplethysmogram among various cardiovascular risk factors in middle-aged men. Hypertension Research. 30, 1211-1218 (2007).

[5] P. G. Gandhi, G. H. R. Rao, The spectral analysis of photoplethysmography to evaluate an independent cardiovascular risk factor. International Journal of General Medicine. 7, 539-547 (2014).

[6] J. Lázaro, E. Gil, J. M. Vergara, P. Laguna, Pulse rate variability analysis for discrimination of sleep-apnea-related decreases in the amplitude fluctuations of pulse photoplethysmographic signal in children. IEEE Journal of Biomedical and Health Informatics. 18, 240-246 (2014)

Address for correspondence:

Marek Żyliński

Institute of Metrology and Biomedical Engineering

Department of Mechatronics

Warsaw University of Technology

Św. Andrzeja Boboli 8

02-525 Warsaw

Poland

zylinski@mchtr.pw.edu.pl 